\title{
Genetic diversity and phylogenetic analysis of Tams1 of Theileria annulata isolates from three continents between 2000 and 2012
}

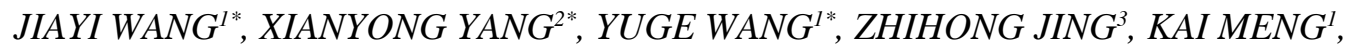 \\ JIANZHU LIU ${ }^{l}$, HUIJUN GUO ${ }^{l}$, RUIXUE XU ${ }^{l}$, ZIGIANG CHENG ${ }^{l}$
}

${ }^{1}$ College of Veterinary Medicine, Research Center for Animal Disease Control Engineering Shandong Province, Shandong Agricultural University, Tai' an 271018, China

${ }^{2}$ Central Hospital of Tai' an City, Tai' an, Shandong, 271018, China

${ }^{3}$ College of Foreign Languages, Shandong Agricultural University, Tai' an 271018, China

"These authors contributed equally to the work.

\begin{abstract}
Theileria annulata, which is part of the Theileria sergenti/Theileria buffeli/Theileria orientalis group, preferentially infects cattle and results in high mortality and morbidity in the Mediterranean, Middle East, and Central Asia. The polypeptide Tams1 is an immunodominant major merozoite piroplasm surface antigen of $T$. annulata that could be used as a marker for epidemiological studies and phylogenetic analysis. In the present study, a total of 155 Tams1 sequences were investigated for genetic diversity and phylogenetic relationships through phylogenetic analysis. Results showed that the Tams 1 sequences were divided into two major groups and that distribution for some isolates also exhibited geographic specificity. As targeting polymorphic genes for parasite detection may result in underestimation of infection, polymerase chain reaction (PCR) assay using two different probes targeting tams-1 genes of these two groups can be more credible. In addition, the direction of the spread of the disease was discovered to be from the Mediterranean or the tropical zone to the Eurasian peninsula, Middle East, Southern Asia, and Africa, particularly for Group 2. A similar occurrence was also found between the Ms1 gene of Theileria lestoquardi and the Tams1 gene of T. annulata, which explains cross-immunogenicity to a certain extent. However, no potential glycosylation site in the Tams1 of T. annulata was found in this study, which illustrated that instead of $N$-glycosylation, other modifications have more significant effects on the immunogenicity of the Tams1 protein.
\end{abstract}

Key words: genetic diversity, phylogenetic analysis, Tams1, Theileria annulata, three continents.

(Centr Eur J Immunol 2014; 39 (4): 476-484)

\section{Introduction}

Various species of Theileria bovine parasites are widespread in Southern Europe, North Africa, and Southern Asia, thus presenting a significant threat to livestock productivity [1]. Among these, the tick-borne protozoan parasite Theileria annulata is the causative agent of lymph proliferative theileriosis, an important disease with high mortality and morbidity. Recently, in an epidemiological survey of bovine Babesia and Theileria parasites, T. annulata has been found to be the most common blood parasite in cattle, buffalo, and sheep populations, which were bred in different geographical locations in Egypt [2]. Theileria annulata parasitizes the reticuloendothelial system and the red blood cells of cattle. The intermediate host then exhibits symptoms such as high fever $\left(40^{\circ} \mathrm{C}\right.$ to $\left.42^{\circ} \mathrm{C}\right)$, depression, cough, runny nose and watery eyes, anemia, and jaundice. Given that the main vectors of ring theileriosis are Hyalommadetritum, $H$. anatolicumanatolicum, $H$. anatolicumexcavatum, $H$. asiaticum, $H$. dromedarii, and $H$. marginatummarginatum [3], the disease typically begins in May, with outbreaks occurring in June and July, and then gradually subsides.

The polypeptide Tams1 elicits a protective response as an immunodominant major merozoite piroplasm surface antigen against the protozoan parasite $T$. annulata [4, 5], and is considered as a candidate for inclusion in a subunit recombinant vaccine [6]. The Tams1-encoding gene has been developed for PCR-based assays, which could use bovine blood samples to detect $T$. annulata infections. Tams1 protein has also been reported as a candidate to develop a diagnostic enzyme-linked immunosorbent assay

Correspondence: Prof. Jianzhu Liu, College of Veterinary Medicine, Research Center for Animal Disease Control Engineering Shandong Province, Shandong Agricultural University, Tai’an 271018, China, e-mail: liujz@sdau.edu.cn 
(ELISA) because it exhibits significant sequence diversity and no geographic specificity [4]. However, sequencing and phylogenetic analyses of the Egyptian T. annulata showed that theTams- 1 sequences are relatively diverse (87.8-100\% identity values), dispersing themselves across several clades in the phylogenetic tree containing sequences from other countries [2]. Moreover, Tams1 diversity has been reported as being generated by the random mutation of nucleotides during asexual reproduction as well as by the selection of changes that confer a biological advantage instead of the differential expression of the members of a gene family $[7,8]$.

Several attempts have been made to analyze the phylogenetic characteristics of the Tams1 gene of T. annulata [8]. However, no distinct classification for all reported Tams1 sequences obtained from three continents (Asia, Africa, and Europe) is yet available. In the present study, 155 complete Tams 1 genes of $T$. annulata occurring over a wide geographical range were first sequenced. Given that the taxonomic status and epidemiology of $T$. annulata remain undefined [9], studying the phylogenetic variability and molecular genetic characterization of Tams 1 will help provide an understanding of the relationship between the molecular evolutionary history of $T$. annulata and the emergence, breakout, and spread of new T. annulata epidemics.

In addition, attempts were made to analyze the phylogenetic diversity and distribution of $T$. annulata. A phylogenetic tree was constructed and analyzed. Comparisons and additional analyses were also performed, including predicting potential glycosylation sites as well as finding volatile regions and evolutionary regular patterns.

\section{Material and methods}

\section{Theileria sampling, DNA extraction, polymerase chain reaction amplification, and sequencing}

A total of 81 adult ticks were collected from 61 cattle in a randomly selected dairy farm in Xinjiang, a northwestern city in China, in 2012. DNA extraction, as well as polymerase chain reaction (PCR) detection and amplification, were performed by Meng et al. [3]. In brief, a previously reported primer set (Forward 5-GTAACCTTTAAAAACGT-3, Reverse 5-CAGTTACGAACATGGGTTT-3) was used to detect Tams1 DNA specifically $[1,11]$. The recombinant plasmid pMD18-T vector (Takara Bio Inc., Japan) was transformed into Escherichia coli TOP10 competent cells after purified DNA fragments were cloned and inserted. Then, the E. coli that was cultured overnight were purified and sent to a private company [Sangon Biotech (Shanghai) Co., Ltd., China] for sequencing. The results were identified by comparing the obtained sequences with the registered sequences in GenBank through BLAST (http://blast.ncbi.
nlm.nih.gov/Blast.cgi). From the clinical isolate, an identical sequence with the Tams 1 gene of $T$. annulata was found (GenBank accession no. JX475044).

\section{Sequence alignment and phylogenetic analysis}

Tams 1 gene sequences published in GenBank were also included. Blasting was performed at the National Center for Biotechnology Information (http://www.ncbi.nlm. nih.gov/). The amplified sequences and Tams1 were both blasted to ensure that all known Tams1 genes of T. annula$t a$ were brought forward. Consequently, a total of 155 isolates were discovered (Table 1) including RefSeq (Turkey, 1), Spain (14), Portugal (5), Italy (5), Tunisia (46), Iran (4), Bahrain (9), Turkey (18), India (7), Mauritania (22), Iraq (7), China (2), Sudan (5), Sri Lanka (3), unknown origin (4), and isolates of T. lestoquardi (4) [11].

Multiple sequence alignments were performed using the ClustalW algorithm [12]. The phylogenetic tree was constructed through the neighbor-joining method [13] and a bootstrap value of 1000 replicates, using the MEGA 5.1 software [14].

\section{Predicting N-glycosylation sites}

Glycosylation sites have an important role in determining the properties of the concerned protein, such as antigenic properties, among others $[15,16]$. Considering that the glycosylation sites of the protein were formed after processing, only potential glycosylation sites were predicted at the amino acid level. In this investigation, referring to the phylogenetic tree, 39 isolates (including XM948626.1, AF214906.1, AF214872.1, AF214920.1, AF214904.1, AF214819.1, AF214840.1, JX648210.1, AF214832.1, AF214852.1, AF214849.1, AF214818.1, AF214898.1, EU563912.1, JX475044, AJ276654.1, EF618726.1, AF214869.1, AF214900.1, GU130193.1, AF214866.1, AF214856.1, AF214835.1, U22888.1, AF214800.1, AF214801.1, AF214812.1, AF214879.1, EF092915.1, AF214825.1, AF214815.1, AB690864.1, AF214805.1, AF214797.1, AF214863.1, AF214875.1, GU130190.1, EF092918.1, FJ159695.1) on behalf of 155 isolates were selected, followed by the submission of the amino acid sequence of the protein to NetNGlyc Server (http://www. cbs.dtu.dk/services/NetNGlyc/) [17], and the prediction of the $\mathrm{N}$-glycosylation sites in the amino acid sequence of the protein.

\section{Results}

Based on the multiple sequence alignments and the neighbor-joining method, the sequence obtained from the clinical isolate and the 154 sequences (including four Ms1 genes from T. lestoquardi) in GenBank of Tams1 isolates derived from the three continents (Asia, Africa and Europe) were assigned to two groups (Fig. 1). Sequences 
Table 1. Origin of Tams1 isolates used in the present study

\begin{tabular}{|c|c|c|c|c|c|c|c|}
\hline No. & Country & Year & Accession no. & No. & Country & Year & Accession no. \\
\hline 1 & Spain & 2000 & AF214813.1 & 42 & India & 2000 & AF214844.1 \\
\hline 2 & Spain & 2000 & AF214816.1 & 43 & Tunisia & 2000 & AF214868.1 \\
\hline 3 & Spain & 2000 & AF214814.1 & 44 & Tunisia & 2000 & AF214866.1 \\
\hline 4 & Spain & 2000 & AF214815.1 & 45 & Mauritania & 2000 & AF214817.1 \\
\hline 5 & Spain & 2000 & AF214827.1 & 46 & Mauritania & 2000 & AF214822.1 \\
\hline 6 & Italy & 2000 & AF214862.1 & 47 & Iraq & 2010 & Gu130194.1 \\
\hline 7 & Italy & 2000 & AF214860.1 & 48 & Iraq & 2010 & Gu130193.1 \\
\hline 8 & Spain & 2000 & AF214809.1 & 49 & Tunisia & 2000 & AF214869.1 \\
\hline 9 & Iran & 2006 & EF092915.1 & 50 & Tunisia & 2000 & AF214871.1 \\
\hline 10 & Tunisia & 2000 & AF214879.1 & 51 & Tunisia & 2000 & AF214870.1 \\
\hline 11 & Tunisia & 2000 & AF214864.1 & 52 & Bahrain & 2000 & AF214796.1 \\
\hline 12 & Tunisia & 2000 & AF214903.1 & 53 & Bahrain & 2007 & EF618728.1 \\
\hline 13 & Tunisia & 2000 & AF214882.1 & 54 & India & 2007 & EF618726.1 \\
\hline 14 & Tunisia & 2000 & AF214865.1 & 55 & Tunisia & 2000 & AF214878.1 \\
\hline 15 & Spain & 2000 & AF214812.1 & 56 & Tunisia & 2000 & AF214877.1 \\
\hline 16 & Spain & 2012 & JX683683.1 & 57 & Tunisia & 2000 & AF214887.1 \\
\hline 17 & Spain & 2006 & Z48739.1 & 58 & Tunisia & 2000 & AF214867.1 \\
\hline 18 & Spain & 1995 & U22888.1 & 59 & Tunisia & 2000 & AF214900.1 \\
\hline 19 & Bahrain & 2000 & AF214802.1 & 60 & Tunisia & 2000 & AF214889.1 \\
\hline 20 & Bahrain & 2000 & AF214795.1 & 61 & Tunisia & 2000 & AF214888.1 \\
\hline 21 & Bahrain & 2000 & AF214799.1 & 62 & Turkey & 2000 & AF214909.1 \\
\hline 22 & Turkey & 2000 & AF214835.1 & 63 & Turkey & 2000 & AF214915.1 \\
\hline 23 & Mauritania & 2000 & AF214851.1 & 64 & Turkey & 2010 & AJ276654.1 \\
\hline 24 & Mauritania & 2000 & AF214856.1 & 65 & Turkey & 2000 & AF214918.1 \\
\hline 25 & Mauritania & 2000 & AF214853.1 & 66 & Turkey & 2008 & XM948626.1 \\
\hline 26 & Mauritania & 2000 & AF214850.1 & 67 & Turkey & 2000 & AF214916.1 \\
\hline 27 & Portugal & 2000 & AF218426.1 & 68 & Mauritania & 2000 & AF214858.1 \\
\hline 28 & Portugal & 2000 & AF218425.1 & 69 & Mauritania & 2000 & AF214846.1 \\
\hline 29 & Portugal & 2000 & AF218429.1 & 70 & Tunisia & 2000 & AF214898.1 \\
\hline 30 & Portugal & 2000 & AF218428.1 & 71 & Tunisia & 2000 & AF214897.1 \\
\hline 31 & Tunisia & 2000 & AF214919.1 & 72 & Mauritania & 2000 & AF214854.1 \\
\hline 32 & Tunisia & 2000 & AF214895.1 & 73 & Mauritania & 2000 & AF214821.1 \\
\hline 33 & Tunisia & 2000 & AF214893.1 & 74 & Mauritania & 2000 & AF214824.1 \\
\hline 34 & Tunisia & 2000 & AF214894.1 & 75 & Mauritania & 2000 & AF214823.1 \\
\hline 35 & Tunisia & 2000 & AF214880.1 & 76 & Mauritania & 2000 & AF214848.1 \\
\hline 36 & Bahrain & 2000 & AF214794.1 & 77 & Mauritania & 2000 & AF214845.1 \\
\hline 37 & Bahrain & 2000 & AF214801.1 & 78 & Mauritania & 2000 & AF214819.1 \\
\hline 38 & Bahrain & 2000 & AF214798.1 & 79 & Tunisia & 2000 & AF214883.1 \\
\hline 39 & Turkey & 2000 & AF214836.1 & 80 & Tunisia & 2000 & AF214881.1 \\
\hline 40 & Turkey & 2000 & AF214837.1 & 81 & Tunisia & 2000 & AF214901.1 \\
\hline 41 & Bahrain & 2000 & AF214800.1 & 82 & Tunisia & 2000 & AF214920.1 \\
\hline
\end{tabular}


Table 1. Cont.

\begin{tabular}{|c|c|c|c|c|c|c|c|}
\hline No. & Country & Year & Accession no. & No. & Country & Year & Accession no. \\
\hline 83 & Tunisia & 2000 & AF214885.1 & 120 & Turkey & 2000 & AF214912.1 \\
\hline 84 & Iran & 2004 & AY672541.1 & 121 & Turkey & 2000 & AF214913.1 \\
\hline 85 & China & 2012 & JX475044.1 & 122 & Iraq & 2010 & GU130191.1 \\
\hline 86 & Turkey & 2000 & AF214917.1 & 123 & Iraq & 2010 & GU130190.1 \\
\hline 87 & Turkey & 2000 & AF214914.1 & 124 & Italy & 2000 & AF214859.1 \\
\hline 88 & Turkey & 2000 & AF214838.1 & 125 & Italy & 2000 & AF214863.1 \\
\hline 89 & Turkey & 2000 & AF214839.1 & 126 & Italy & 2000 & AF214861.1 \\
\hline 90 & Mauritania & 2000 & AF2w14857.1 & 127 & Bahrain & 2000 & AF214797.1 \\
\hline 91 & Mauritania & 2000 & AF214820.1 & 128 & Spain & 2000 & AF214808.1 \\
\hline 92 & China & 2008 & EU593912.1 & 129 & Spain & 2000 & AF214807.1 \\
\hline 93 & India & 2000 & AF214842.1 & 130 & Spain & 2000 & AF214805.1 \\
\hline 94 & Mauritania & 2000 & AF214818.1 & 131 & Spain & 2000 & AF214804.1 \\
\hline 95 & Mauritania & 2000 & AF214849.1 & 132 & Spain & 2000 & AF214803.1 \\
\hline 96 & Mauritania & 2000 & AF214855.1 & 133 & Spain & 2000 & AF214810.1 \\
\hline 97 & Sudan & 2000 & AF214834.1 & 134 & Spain & 2000 & AF214811.1 \\
\hline 98 & Sudan & 2000 & AF214831.1 & 135 & Spain & 2000 & AF214806.1 \\
\hline 99 & Sudan & 2000 & AF214832.1 & 136 & Sri Lanka & 2012 & AB690865.1 \\
\hline 100 & Sudan & 2000 & AF214833.1 & 137 & Sri Lanka & 2012 & AB690864.1 \\
\hline 101 & Sudan & 2000 & AF214830.1 & 138 & Sri Lanka & 2012 & AB690863.1 \\
\hline 102 & India & 2000 & AF214843.1 & 139 & Iran & 2006 & EF092916.1 \\
\hline 103 & South India & 2012 & JX648210.1 & 140 & Iran & 2000 & AF004775.2 \\
\hline 104 & India & 2000 & AF214841.1 & 141 & Iran & 1999 & AJ006447.1 \\
\hline 105 & India & 2000 & AF214840.1 & 142 & Iran & 1999 & AJ006448.1 \\
\hline 106 & Mauritania & 2000 & AF214847.1 & 143 & Iraq & 2010 & FJ159695.1 \\
\hline 107 & Tunisia & 2000 & AF214899.1 & 144 & Tunisia & 2000 & AF214876.1 \\
\hline 108 & Tunisia & 2000 & AF214872.1 & 145 & Tunisia & 2000 & AF214874.1 \\
\hline 109 & Turkey & 2000 & AF214911.1 & 146 & Tunisia & 2000 & AF214875.1 \\
\hline 110 & Tunisia & 2000 & AF214873.1 & 147 & Tunisia & 2000 & AF214896.1 \\
\hline 111 & Tunisia & 2000 & AF214886.1 & 148 & Tunisia & 2000 & AF214892.1 \\
\hline 112 & Tunisia & 2000 & AF214906.1 & 149 & Tunisia & 2000 & AF214890.1 \\
\hline 113 & Tunisia & 2000 & AF214907.1 & 150 & Tunisia & 2000 & AF214891.1 \\
\hline 114 & Tunisia & 2000 & AF214905.1 & 151 & Turkey & 2000 & AF214908.1 \\
\hline 115 & Tunisia & 2000 & AF214884.1 & 152 & Mauritania & 2000 & AF214852.1 \\
\hline 116 & Iran & 2006 & EF092919.1 & 153 & Tunisia & 2000 & AF214904.1 \\
\hline 117 & Iran & 2006 & EF092918.1 & 154 & Tunisia & 2000 & AF214902.1 \\
\hline 118 & Iraq & 2010 & GU130192.1 & 155 & Iraq & 2010 & GU130189.1 \\
\hline 119 & Turkey & 2000 & AF214910.1 & & & & \\
\hline
\end{tabular}




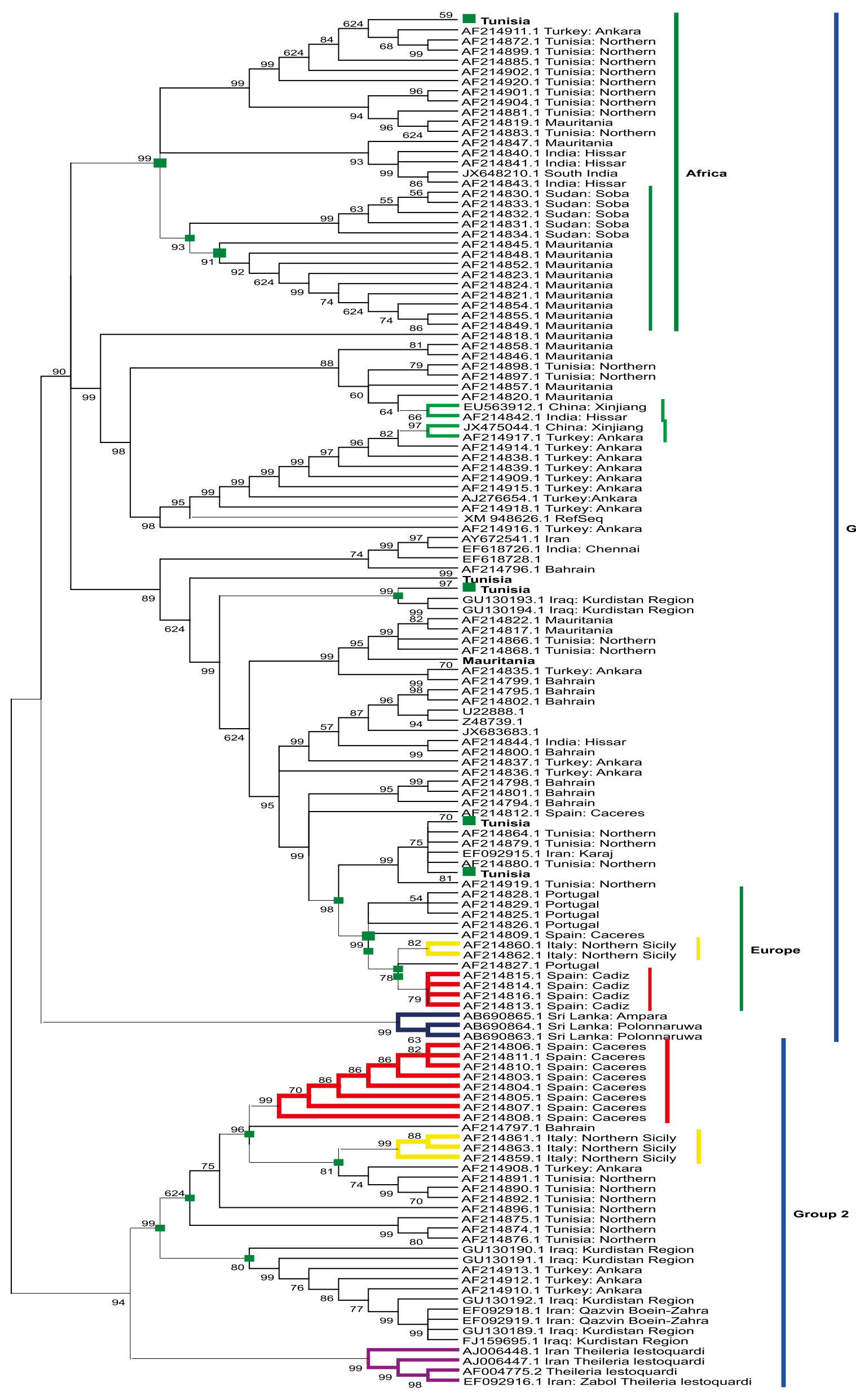




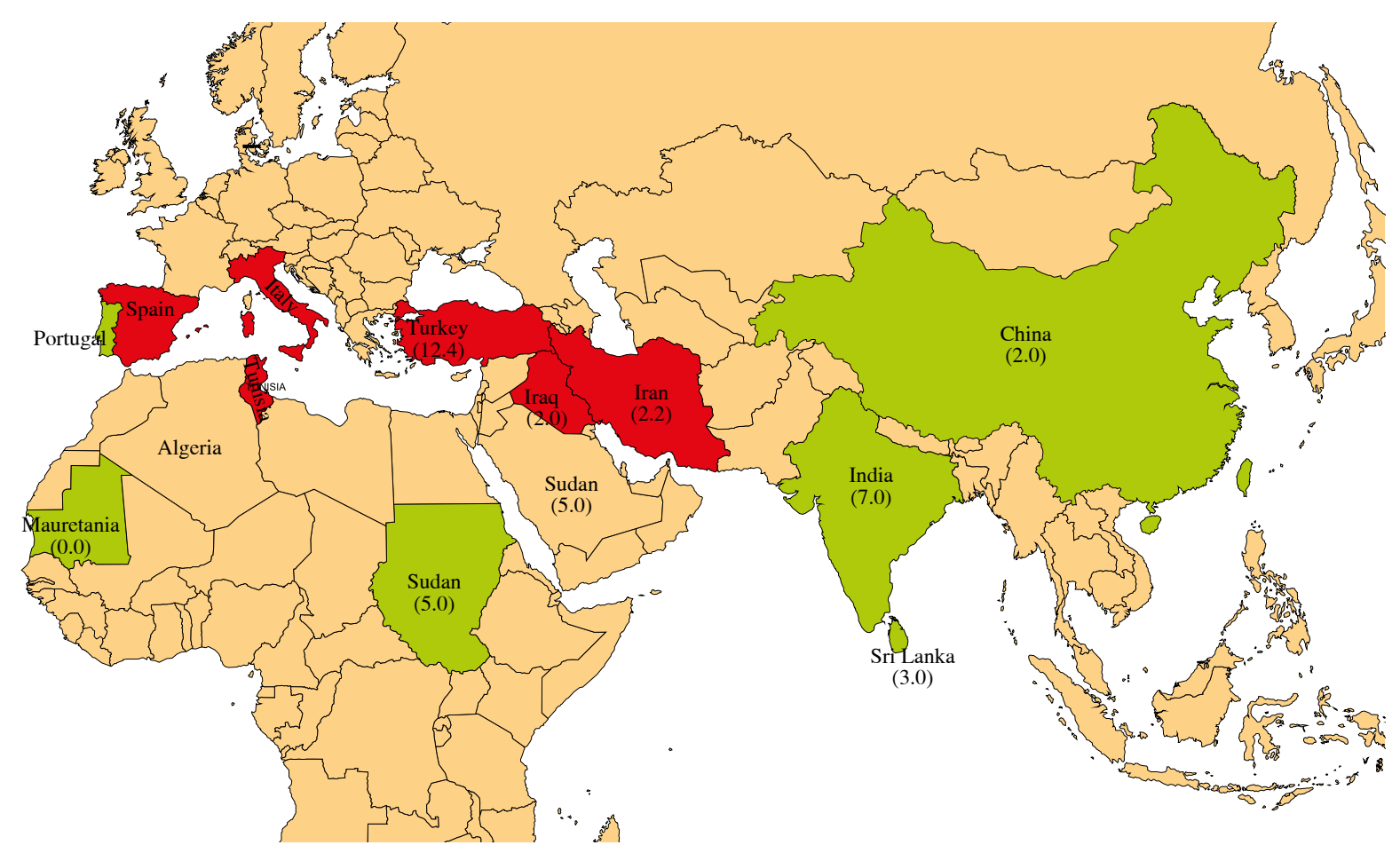

In the green parts, only T. annulata of group two can be found, while in the red areas T. annulata of both groups can be found. And the numbers in the brackets means isolate number in group 1 and isolate number in group 2.

Fig. 2. The geographical distribution of Theileria annulata

from Spain, Italy, Tunisia, Iran, Bahrain, Turkey and Iraq were found in both groups, while in Portugal, India, Mauritania, China, Sudan and Sri Lanka, sequences were only found in Group 1. In the phylogenetic tree, isolates from the same continents (Africa and Europe) got-together in Group 1 (Fig. 2). The N-glycosylation sites' prediction by online software showed that no potential glycosylation sites were found.

\section{Discussion}

The phylogenetic tree constructed with 155 Tams 1 gene sequences (including four Ms1 genes from $T$. lestoquardi) shows that $T$. annulata isolates were divided into two major groups, which were called Groups 1 and 2. This discovery was very important and significant, as almost every research about Tams1 would bring up some similar guesses [18]. Habibi [19] classified 17 Tams1 gene sequences into 2 clusters. However, classifying all reported Tams 1 sequences obtained from three continents (Asia, Africa, and Europe) has not yet been performed.

Several studies found that the Tams1 gene is highly polymorphic, raising questions concerning the suitability of Tams 1 gene-targeted primers to detect all $T$. annulata isolates [1]. And especially in clinical practice, as targeting polymorphic genes for parasite detection may result in un-
Table 2. Numbers of Theileria annulata isolates in groups 1 and 2 in each country

\begin{tabular}{lcccc}
\hline \multicolumn{1}{c}{ Country } & Group 1 & Year & Group 2 & Year \\
\hline Spain & 6 & 2000 & 8 & 2000 \\
\hline Portugal & 5 & 2000 & 0 & \\
\hline Italy & 2 & 2000 & 3 & 2000 \\
\hline Tunisia & 39 & 2000 & 7 & 2000 \\
\hline Iran & 1 & 2004 & 2 & 2006 \\
\hline & 1 & 2006 & & 2000 \\
\hline Bahrain & 8 & 2000 & 1 & 2000 \\
\hline Turkey & 11 & 2000 & 4 & \\
\hline & 1 & 2010 & & \\
\hline India & 5 & 2000 & 0 & \\
\hline & 2 & 2007 & & \\
\hline Sudan & 1 & 2012 & & \\
\hline Sri Lanka & 3 & 2000 & 0 & \\
\hline Mauritania & 22 & 2010 & 5 & 010 \\
\hline Iraq & 2 & 2001 & & \\
\hline China & 1 & 2012 & & \\
\hline & 5 & 2000 & 0 & \\
\hline & 1 & & & \\
\hline & 2012 & & \\
\hline
\end{tabular}

Fig. 1. Phylogenetic tree for the Tams 1 gene of the 155 Theileria annulata isolates 

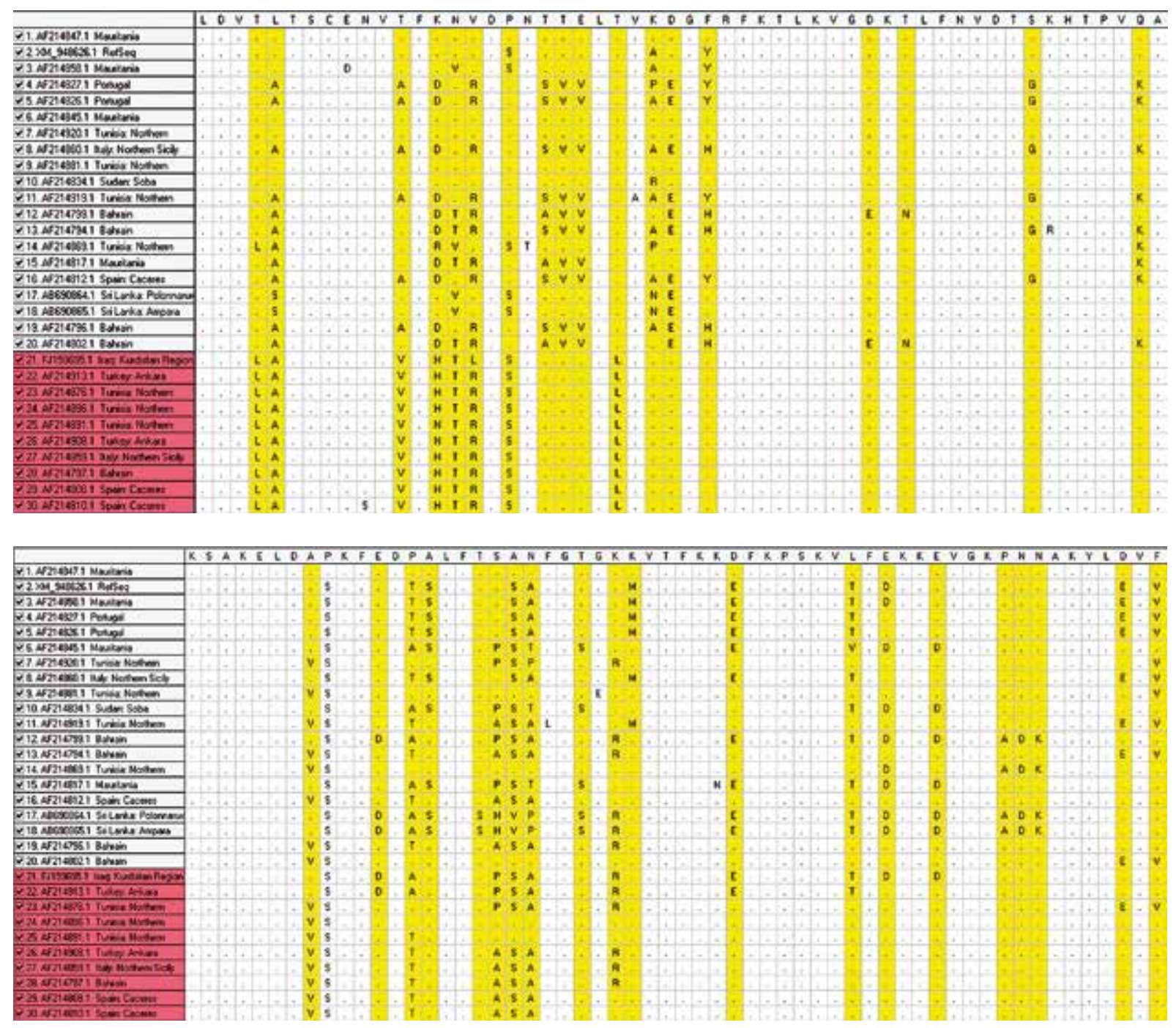

The first 20 sequences were from Group 1, while the last 10 sequences marked in red were from Group 2. The volatile sites and regions in Tams 1 gene were marked in yellow in the figure.

Fig. 3. Analysis and comparison of amino acid mutations in Tams1

derestimation of infection, PCR assay using two different probes targeting tams-1 genes of these two groups, for example the primers (Forward 5-GTAACCTTTAAAAACGT-3, Reverse 5-CAGTTACGAACATGGGTTT-3) [3] and primers (Forward 5-ATGTGTCCAGGACCACCC-3, Reverse 5-GGGTTTTAAAGGAAGTAAAGG-3) [4], can be more credible than just relying on one primer pair [18]. The sequences from Spain, Italy, Tunisia, Iran, Bahrain, Turkey, and Iraq were found in both groups, whereas the sequences from Portugal, India, Mauritania, China, Sudan, and Sri Lanka were only found in Group 1. In this group, isolates from the same continents (Africa and Europe) were obviously clustered in the phylogenetic tree (Fig. 1).
Previous studies have reported that no geographic specificity was observed and nearly identical sequences occurred in different geographic areas. Moreover, a panmictic (from panmixia, that is, the ability of individuals in a population to move freely within their habitat, and thus breed with other members of the population) population structure was suggested by the results of several studies [4]. In the present study, however, the comparison of the Tams 1 gene sequences obtained from three continents revealed that particular sequence types belong to definitive regions, such as Sudan, Sri Lanka, and so on (marked in dark green and purple in Fig. 1). Nearly no identical Tams1 sequence was found in widely separated regions. 
By referring to Fig. 2 and Table 2, the direction of the spread of the disease, particularly for Group 2, can be speculated. Based on the time (as the recording time reflects the isolates' outbreak time in some degree) and distribution, the following conclusions can be drawn. Isolates from both groups were found in the European peninsula in 2000 , in Iran in 2006, and in Iraq in 2010. However, only Group 1 isolates were found in China, India, and Sri Lanka. Group 2 isolates eventually spread to Iraq. The overall spread direction was from the Mediterranean or the tropical zone to the Eurasian peninsula, Middle East, Southern Asia, and Africa.

Theileria annulata has the ability to transform the leukocytes of host animals and been called transforming Theileria. The evolution of the transforming Theileria has been accompanied by drastic changes in its genetic makeup, such as acquisition or expansion of gene families, which are thought to play critical roles in the transformation and the immune escapement of host cells [20]. Immune escape in Theileria is facilitated by genetic diversity in its antigenic determinants, which potentially results in a loss of $\mathrm{T}$ cell receptor recognition in its host. And in the case of AsnXaa-Ser/Thrsequons (incl. Asn-Pro-Ser/Thr), no potential glycosylation site was discovered. This positive verified the suspect of Katzer in 2002 that there were no N-glycosylation sites on Tams1 genes [21]. Consequently, Tams1 protein easily combined with the antibody as a stable protein antigen. These results indicate that compared with $\mathrm{N}$-glycosylation, other modifications have more significant effects on the immunogenicity of the Tams 1 protein.

Cows in the aforementioned three continents have been reported to have been infected with mixed populations of geographically variable Theileria parasites [9, 23]. Cross-immunity studies conducted by Leeman [22] suggested that partial cross-immunity from $T$. annulata to T. lestoquardi, and vice versa, developed in sheep, thus indicating a close relationship among parasite species. As the tree may not be a true representation of the evolutionary relationship of these sequences/species, however, Tams 1 sequences for $T$. annulata would be more likely to cluster together than with sequences from a distinct species. Coincidentally, four T. lestoquardi Ms1 gene isolates (Query cover: $86 \%$ to $95 \%$, ident: $85 \%$ to $86 \%$ ) were obtained by blasting the Tams1 gene of T. annulata and were included in Group 2 through phylogenetic assay. This finding provides evidence of a significant similarity between the Ms1 gene of T. lestoquardi and the Tams1 gene of T. annulata, thus explaining the immunogenicity of the Tams 1 protein to a certain extent.

Given that it enables evasion from host immune response, diversity is generally believed to be a positively selected result [24]. In Fig. 3, several variable areas were marked. These areas represent the generation of Tams1 diversity that enabled $T$. annulata to escape from host immunity. This finding provides valuable information on the antigenic structure of $T$. annulata and may be helpful in designing vaccines.

\section{Conclusions}

Tams1, as an immunodominant surface antigen, has been used to investigate genetic diversity and vaccine purposes for antigenicity [4]. In the present study, all 155 Tams 1 isolates were classified into two major groups in the phylogenetic tree, which explained why PCR assay using one primer pair targeting tams- 1 gene failed to detect infection of some animals. Geographic specificity was observed when comparing Tams1 gene sequences. In addition, based on the geographic distribution and recording time of T. annulata, the spread direction of the disease, particularly for Group 2, was hypothesized to be from the Mediterranean or the tropical zone to the Eurasian peninsula, Middle East, Southern Asia, and Africa. A similarity on immunodominant major surface antigen gene was also found between the Ms1 gene of T. lestoquardi and the Tams 1 gene of T. annulata, which explains cross-immunogenicity to a certain extent. However, no potential glycosylation site was found in this study, thus instead of $N$-glycosylation, other modifications have been hypothesized to have more significant effects on the immunogenicity of the Tams1 protein.

The authors declare no conflict of interest.

This work was supported by Shandong Modern Agricultural Technology \& Industry System (No. SDAIT-13-011-04), the Natural Science Foundation of Shandong Province (ZR2012CQ001), the Research Fund for the Doctoral Program of Higher Education (20123702120008).

\section{References}

1. Santos M, Soares R, Costa P, et al. (2013): Revisiting the Tams1-encoding gene as a species-specific target for the molecular detection of Theileria annulata in bovine blood samples. Ticks Tick Borne Dis 4: 72-77.

2. Elsify A, Sivakumar T, Nayel M, et al. (2014): An epidemiological survey of bovine Babesia and Theileria parasites in cattle, buffaloes, and sheep in Egypt. Parasitol Int [Epub ahead of print].

3. Meng K, Li Z, Wang Y, et al. (2014): PCR-based detection of Theileria annulata in Hyalomma asiaticum ticks in northwestern China. Ticks Tick Borne Dis 5: 105-106.

4. Gubbels MJ, Katzer F, Hide G, et al. (2000): Generation of a mosaic pattern of diversity in the major merozoite-piroplasm surface antigen of Theileria annulata. Mol Biochem Parasitol 110: 23-32.

5. Gharbi M, Darghouth MA, Weir W, et al. (2011): Primeboost immunisation against tropical theileriosis with two 
parasite surface antigens: Evidence for protection and antigen synergy. Vaccine 29: 6620-6628.

6. Boulter N, Hall R (1999): Immunity and vaccine development in the bovine theilerioses. Adv Parasitol 44: 41-97.

7. Weir W, Karagenç T, Baird M, et al. (2010): Evolution and diversity of secretome genes in the apicomplexan parasite Theileria annulata. BMC Genomics 11: 42.

8. Esmaelizad M, Niaraki S J, Fesharaki R H (2011): Molecular and phylogenetic analysis of the partial tams1 gene sequence of a vaccine strain of Theileria annulata. Braz Arch Biol Tech 54: 1109-1116.

9. Kim SJ, Tsuji M, Kubota S, et al. (1998): Sequence analysis of the major piroplasm surface protein gene of benign bovine Theileria parasites in East Asia. Int J Parasitol 28: 1219-1227.

10. Katzer F, McKellar S, Kirvar E, Shiels B (1998): Phylogenetic analysis of Theileria and Babesia equi in relation to the establishment of parasite populations within novel host species and the development of diagnostic tests. Mol Biochem Parasitol 95: 33-44.

11. Pan W, Gao Y, Qin L, et al. (2012): Genetic diversity and phylogenetic analysis of glycoprotein GP85 of ALV-J isolates from Mainland China between 1999 and 2010: coexistence of two extremely different subgroups in layers. Vet Microbiol 156: 205-212.

12. Thompson JD, Higgins DG, Gibson TJ (1994): CLUSTAL $\mathrm{W}$ : improving the sensitivity of progressive multiple sequence alignment through sequence weighting, position-specific gap penalties and weight matrix choice. Nucleic Acids Res 22: 4673-4680.

13. Saitou N, Nei M (1987): The neighbor-joining method: a new method for reconstructing phylogenetic trees. Mol Biol Evol 4: 406-425.

14. Tamura K, Dudley J, Nei M, Kumar S (2007): MEGA4: molecular evolutionary genetics analysis (MEGA) software version 4.0. Mol Biol Evol 24: 1596-1599.

15. Chen XL, Shi T, Yang J, et al. (2014): N-glycosylation of effector proteins by an $\alpha-1,3$-mannosyltransferase is required for the rice blast fungus to evade host innate immunity. Plant Cell 26: 1360-1376.

16. He P, Srikrishna G, Freeze HH (2014): An N-glycosylation deficiency reduces ICAM-1 induction and impairs inflammatory response. Glycobiology 24: 392-398.

17. Auguste AJ, Carrington CV, Forrester NL, et al. (2014): Characterization of a novel Negevirus and a novel Bunyavirus isolated from Culex (Culex) declarator mosquitoes in Trinidad. J Gen Virol 95: 481-485.

18. Ghoneim AM, El-Fayomy AO (2014): Targeting tams-1 gene results in underestimation of Theileria annulata infection in diseased cattle in Egypt. Acta Parasitol 59: 85-90.

19. Habibi G (2013): A comparative phylogenetic analysis of Theileria spp. by using two " $18 \mathrm{~S}$ ribosomal RNA" and "Theileria annulata merozoite surface antigen" gene sequences. Arch Razi 68: 47-52.

20. Sivakumar T, Hayashida K, Sugimoto C, Yokoyama N (2014): Evolution and genetic diversity of Theileria. Infect Genet Evol 27: 250-263.

21. Katzer F, McKellar S, Ferguson MA, et al. (2002): A role for tertiary structure in the generation of antigenic diversity and molecular association of the Tams1 polypeptide inTheileria annulata. Mol Biochem Parasitol 122: 55-67.

22. Thumbi SM, Bronsvoort BM, Poole EJ, et al. (2014): Parasite co-infections and their impact on survival of indigenous cattle. PLoS One 9: e76324.
23. Leemans I, Brown D, Hooshmand-Rad P, et al. (1999): Infectivity and cross-immunity studies of Theileria lestoquardi and Theileria annulata in sheep and cattle: I. In vivo responses. Vet Parasitol 82: 179-192.

24. Cooke CL, Huff JL, Solnick JV (2005): The role of genome diversity and immune evasion in persistent infection with Helicobacter pylori. FEMS Immunol Med Microbiol 45: 11-23. 